\title{
Atomic levels in superstrong magnetic fields and $D=2$ QED of massive electrons: screening
}

\author{
M.I. Vysotsky \\ ITEP, Moscow
}

April 19, 2022

\begin{abstract}
The photon polarization operator in superstrong magnetic fields induces the dynamical photon "mass" which leads to screening of Coulomb potential at small distances $z \ll 1 / m, m$ is the mass of an electron. We demonstrate that this behaviour is qualitatively different from the case of $D=2 \mathrm{QED}$, where the same formula for a polarization operator leads to screening at large distances as well. Because of screening the ground state energy of the hydrogen atom at the magnetic fields $B \gg m^{2} / e^{3}$ has the finite value $E_{0}=-m e^{4} / 2 \ln ^{2}\left(1 / e^{6}\right)$.
\end{abstract}

\section{Introduction}

The Larmour radius of the electron orbit $a_{H}=1 / \sqrt{e B}$ is much smaller than Bohr atomic radius $a_{B}=1 /\left(m e^{2}\right)$ for homogenius magnetic fields $B \gg m^{2} e^{3}$ (we are using Gauss system of units, where $e^{2}=\alpha=1 / 137$; also in all formulas $\hbar=c=1$ ). It is natural to look for the atomic energy levels in such strong magnetic fields studing the influence of Coulomb potential on the electrons occupying Landau levels [1]. A strong magnetic field confines an electron in the transverse direction while in the longitudinal direction an electron is bound by the weak Coulomb field of a nucleus. The cigar-shape wave function of an electron is formed with transverse size which equals Larmour 
radius and longitudinal size which is by $\ln \left(a_{B}^{2} / a_{H}^{2}\right) \equiv \ln \left(B / m^{2} e^{3}\right)$ smaller than Bohr radius. The ground state energy is larger than Rydberg constant by the square of the same logarithm: $E_{0}=-\left(m e^{4} / 2\right) \ln ^{2}\left(B / m^{2} e^{3}\right)$. One can easily get this logarithmic factor from the fact that in one-dimensional Coulomb potential energy diverges logarithmically at small distances. The divergency is regularized at the longitudinal distances which equals $a_{H}$, where one-dimensional motion converts to a three-dimensional one. Atomic levels in such strong magnetic fields were found numerically in 2] (see also [3, 4]).

Our purpose is to understand the behaviour of the energy levels with the growth of a magnetic field. The point is that at superstrong magnetic fields $B \gtrsim m^{2} / e^{3}$ the polarization operator insertions into the photon propagator induce the dynamic photon "mass" $m_{\gamma}^{2} \approx e^{3} B$ [5, 6]. One would expect that the photon mass should screen Coulomb potential and shift energies of the atomic levels found in tree approximation.

Dirac equation spectrum in a constant homogenious magnetic field looks like [7]:

$$
\varepsilon_{n}^{2}=m^{2}+p_{z}^{2}+(2 n+1) e B+\sigma e B
$$

where $n=0,1,2, \ldots, \sigma= \pm 1$ and the field is directed along axis $z$ 1 In the magnetic fields we are interested in $\varepsilon_{n} \gtrsim m / e$, and electrons are ultrarelativistic. The only exception is the lowest Landau level (LLL) which has $n=0, \sigma=-1$. The energy of LLL electron equals its mass for $p_{z}=0$ and the consideration of the nonrelativistic electron motion along $z$ axis is selfconsistent. LLL is interesting both practically and theoretically. An analog of the critical electric field $E_{c r}=m^{2} / e$ is the magnetic field $B_{0}=m^{2} / e=4.4 \cdot 10^{13}$ gauss. Two orders larger superstrong fields $B \gtrsim m^{2} / e^{3}$ can exist at special neutron stars named magnetars. The temperature of an outer magnetar layer is not enough to populate the excited Landau levels and one can observe the transitions among the states to which LLL is splitted at the electric field of the nucleus. Freezing of the ground state energy in the superstrong magnetic fields discussed in the paper leads to the upper bound on the spectra of photons radiated from magnetars. 2 To study the stability of the huge magnetic fields [9] one should also know the energy of the ground state as a function of a field.

So we are studying the energies of the states to which LLL splits in the

\footnotetext{
${ }^{1}$ This spectrum with the substitution of $2 n+1+\sigma$ by $2 j, j=0,1,2, \ldots$ was found by I.I. Raby 8$]$.

${ }^{2}$ I am grateful to S.I. Blinnikov for the discussions of magnetar physics.
} 
presence of an atomic nucleus.

Since the electron at LLL moves along $z$ axis we will study in section 2 QED at $D=2$ : the behaviour of electrons in two-dimensional space-time. The coupling constant $g$ has dimension of mass, so Coulomb potential as a function of $|z|$ depends on two dimensionfull parameters: $g$ and electron mass $m$. We will obtain the approximate analytical formula for Coulomb potential in $d=D-1=1$ which takes into account the photon polarization operator. We will see that for large $g$ (or small $m$ ) $g \gg m$ Coulomb potential is screened.

In section 3 we will consider the physical case, $D=4$ QED. The analog of the coupling constant squared $g^{2}$ in the real world is the product $e^{3} B$. The polarization operator in the magnetic fields $B \gg m^{2} / e$ at $k_{\|}^{2}\left(\equiv k_{z}^{2}\right) \ll e B$ practically coincides with the one obtained in section 2 [12]. Nevertheless the screening at large distances $|z| \gg 1 / m$ does not occur: at $|z| \gg 1 / m$ we get a purely Coulomb potential $\Phi(z)=e /|z|$. The screening occurs at small distances, and its influence on the ground state energy is determined in section 4 . The results similar to those presented in sections 3 and 4 were obtained in [10] with the help of the numerical calculations.

\section{$2 \quad D=2$ QED: screening}

Coulomb potential in the coordinate space could be obtained by the Fourier transformation of the 00-component of the photon propagator in momentum representation at momentum $k_{\mu}=\left(0, k_{\|}\right)$. We designate the space-like component of momentum by $k_{\|}$, which will be natural in the case $d=3$, see below. The series of Feynman diagrams for the photon proparator is shown in Fig. 1 which corresponds to the following equations:

$$
\Phi(\bar{k}) \equiv A_{0}(\bar{k})=\frac{4 \pi g}{\bar{k}^{2}} ; \quad \boldsymbol{\Phi} \equiv \mathbf{A}_{0}=D_{00}+D_{00} \Pi_{00} D_{00}+\ldots
$$




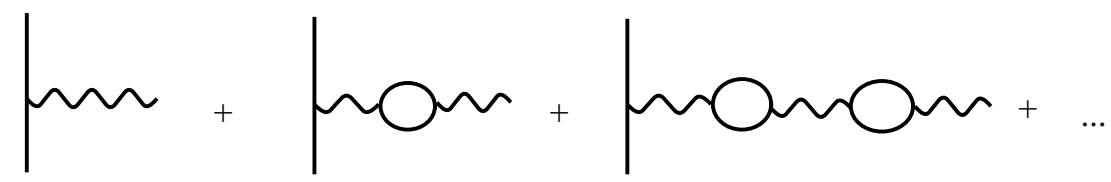

Fig 1. Modification of the Coulomb potential due to the dressing of the photon propagator.

Summing the series we get:

$$
\boldsymbol{\Phi}(k)=-\frac{4 \pi g}{k^{2}+\Pi\left(k^{2}\right)}, \quad \Pi_{\mu \nu} \equiv\left(g_{\mu \nu}-\frac{k_{\mu} k_{\nu}}{k^{2}}\right) \Pi\left(k^{2}\right),
$$

where $\Pi_{\mu \nu}$ is the photon polarization operator at one loop. Instead of calculating the fermion loop we can take an expression for $\Pi$ obtained in the dimensional regularization method [11], substitute $D=2$ in it and divide it by two, because in two dimensions the traces of $\gamma$-matrices are proportional to 2 instead of 4 :

$$
\begin{gathered}
\Pi\left(k^{2}\right)=4 g^{2}\left[\frac{1}{\sqrt{t(1+t)}} \ln (\sqrt{1+t}+\sqrt{t})-1\right] \equiv-4 g^{2} P(t), \\
t \equiv-k^{2} / 4 m^{2}-
\end{gathered}
$$

- a well-known result; for example see [12]. 3

Taking $k=\left(0, k_{\|}\right), k^{2}=-k_{\|}^{2}$ for the Coulomb potential in the coordinate representation we get:

$$
\boldsymbol{\Phi}(z)=4 \pi g \int_{-\infty}^{\infty} \frac{e^{i k_{\|} z} d k_{\|} / 2 \pi}{k_{\|}^{2}+4 g^{2} P\left(k_{\|}^{2} / 4 m^{2}\right)},
$$

and the potential energy for the charges $+g$ and $-g$ is finally:

$$
V(z)=-g \Phi(z)
$$

The calculation of $\boldsymbol{\Phi}(z)$ would be simplified if we were interested in the correction to the potential $\sim g^{2}$. Expanding denominator of (44) and taking into account the first two terms we would deform the integration contour in the plane of complex $k_{\|}$in such a way, that the integration result will

\footnotetext{
${ }^{3}$ It was demonstrated in 12 that in strong magnetic fields photon polarization operator is dominated by the electron from LLL and is essentially given by the $D=2$ expression.
} 
be given by the residue at $k_{\|}=0$ and integration of discontinuity of $P\left(k_{\|}^{2}\right)$ which equals the imaginary part of it. This is analogous to what is done in the textbook [13] when the Uehling-Serber correction to Coulomb potential in $d=3$ is calculated. However to obtain the photon mass we should take into account the whole infinite series - mass is not generated in a finite order of the perturbation theory. Discontinuity of the integrand of (4) is not equal to $\operatorname{Im} P$ and the simplification of the integration does not occur.

Asymptotics of $P(t)$ are:

$$
P(t)=\left\{\begin{array}{ll}
\frac{2}{3} t, & t \ll 1 \\
1, & t \gg 1
\end{array} .\right.
$$

Let us take as an interpolating formula for $P(t)$ the following expression:

$$
\bar{P}(t)=\frac{2 t}{3+2 t} .
$$

We have checked that the accuracy of this approximation is not worse than $10 \%$ for the whole interval of $t$ variation, $0<t<\infty$. Substituting(7) in (4) we easily perform the integration:

$$
\begin{aligned}
\boldsymbol{\Phi}(z) & =4 \pi g \int_{-\infty}^{\infty} \frac{e^{i k_{\|} z} d k_{\|} / 2 \pi}{k_{\|}^{2}+4 g^{2}\left(k_{\|}^{2} / 2 m^{2}\right) /\left(3+k_{\|}^{2} / 2 m^{2}\right)}= \\
& =\frac{4 \pi g}{1+2 g^{2} / 3 m^{2}} \int_{-\infty}^{\infty}\left[\frac{1}{k_{\|}^{2}}+\frac{2 g^{2} / 3 m^{2}}{k_{\|}^{2}+6 m^{2}+4 g^{2}}\right] e^{i k_{\|} z} \frac{d k_{\|}}{2 \pi}= \\
& =\frac{4 \pi g}{1+2 g^{2} / 3 m^{2}}\left[-\frac{1}{2}|z|+\frac{g^{2} / 3 m^{2}}{\sqrt{6 m^{2}+4 g^{2}}} \exp \left(-\sqrt{6 m^{2}+4 g^{2}}|z|\right)\right] .
\end{aligned}
$$

In the case of heavy fermions $(m \gg g)$ the potential is given by the tree level expression; the corrections are suppressed as $g^{2} / \mathrm{m}^{2}$ :

$$
\left.\boldsymbol{\Phi}(z)\right|_{m \gg g}=-2 \pi g|z|\left(1+O\left(\frac{g^{2}}{m^{2}}\right)\right) .
$$

In case of light fermions $(m \ll g)$ the second term which describes Yukawa potential in $d=1$ dominates at the distances $|z|<(1 / \mathrm{g}) \ln (\mathrm{g} / \mathrm{m})$. At larger distances the first term dominates; a coupling constant is suppressed by the factor $3 m^{2} / 2 g^{2}$ with respect to the tree level expression:

$$
\left.\boldsymbol{\Phi}(z)\right|_{m \ll g}= \begin{cases}\pi e^{-2 g|z|} & , \quad z \ll \frac{1}{g} \ln \left(\frac{g}{m}\right) \\ -2 \pi g\left(\frac{3 m^{2}}{2 g^{2}}\right)|z| & , \quad z \gg \frac{1}{g} \ln \left(\frac{g}{m}\right) .\end{cases}
$$


The dependence of the potential energy of the two opposite charges (5) on the distance between them is shown in Figure 24

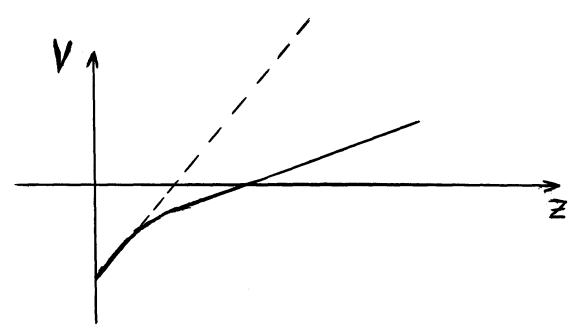

Fig. 2. The potential energy of two opposite charges in $D=2 Q E D$ in the case $g \gg m$. The dashed line shows $V(z)$ for $g \ll m$.

In case $m=0$ a linear term disappears and the potential is determined by the photon with the mass $m_{\gamma}=2 g$ exchange (the Schwinger model: $D=2$ QED with massless fermions [14]). For massive fermions at the distances larger than $\ln (\mathrm{g} / \mathrm{m}) / \mathrm{g}$ we obtain a linear potential with the coupling constant diminished by the factor $3 m^{2} / 2 g^{2}$.

\section{$3 \quad D=4$ QED in a strong magnetic field: screen- ing at $|z|<1 / m$}

In order to derive the potential of the pointlike charge in the realistic case of $D=4$ QED with $d=3$ space-like dimensions in the external magnetic field we should know the expression for a polarization operator in this field. There are many papers where the polarization operator in the external homogeneous field was calculated, see [15] - [17]. The expression for the polarization operator radically simplifies in the magnetic field which is so strong, that the Landau levels spacing is considerably larger than the electron mass, $B \gg$ $B_{0}=m^{2} / e$ and at the longitudinal (parallel to the magnetic field) photon momentum $k_{\|}^{2} \ll e B$, see Eqs.(1.19), (1.22) and (5.2) in [17]. With the help of these formulas we get:

$$
\boldsymbol{\Phi}(k)=-\frac{4 \pi e}{k^{2}+\chi_{2}\left(k^{2}\right)}=
$$

\footnotetext{
${ }^{4}$ I am grateful to A.V. Smilga who noted privately that in the case of light fermions in $D=2$ QED a massive pole in a photon propagator emerges.
} 


$$
=\frac{4 \pi e}{\left(k_{\|}^{2}+k_{\perp}^{2}\right)\left(1+\frac{\alpha}{3 \pi} \ln \left(\frac{2 e B}{m^{2}}\right)\right)+\frac{2 e^{3} B}{\pi} \exp \left(-\frac{k_{\perp}^{2}}{2 e B}\right) P\left(\frac{k_{\|}^{2}}{4 m^{2}}\right)},
$$

where $k=\left(0, k_{x}, k_{y}, k_{z}\right), k_{\perp}^{2}=k_{x}^{2}+k_{y}^{2}, k_{z}=k_{\|}$and the magnetic field is directed parallel to the $z$ axis. This formula is very similar to the analogous formulas from the previous section, see Eqs. (2), (3). The difference is in an extra small term $\sim \alpha \ln \left(e B / m^{2}\right)$, which we will not take into account in the future consideration, and in the factor $2 e^{3} B / \pi \exp \left(-\frac{k_{\perp}^{2}}{2 e B}\right)$ which substitutes $4 g^{2}$. The dependence of function $P$ on $k_{\|}^{2}$ is the same as that in the case of $D=2$ QED. There is also an extra term $k_{\perp}^{2}$ in the denominator and to obtain the potential in the coordinate representation we should integrate over $k_{\perp}$ as well.

With the help of interpolating formula $P(t)$ from section 2 we obtain:

$$
\boldsymbol{\Phi}(z)=4 \pi e \int \frac{e^{i k_{\|} z} d k_{\|} d^{2} k_{\perp} /(2 \pi)^{3}}{k_{\|}^{2}+k_{\perp}^{2}+\frac{2 e^{3} B}{\pi} \exp \left(-k_{\perp}^{2} /(2 e B)\right)\left(k_{\|}^{2} / 2 m^{2}\right) /\left(3+k_{\|}^{2} / 2 m^{2}\right)},
$$

where the integration is performed in the cylindrical coordinates and we are looking for the potential along axis $z$, since it is the potential which bounds an electron in the atom.

We manage to find the asymptotic behaviour of $\boldsymbol{\Phi}(z)$ for $z$ much larger and much smaller than Compton wave length of the electron. For large distances $|z| \gg \frac{1}{m}$ in the integral (12) the region $\left|k_{\|}\right| \ll m$ is important and for the magnetic field $B \gg B_{0}$ we get $k_{\|}^{2} \ll e B$ and the expression for $P$ we are using is correct. For small $\left|k_{\|}\right|$we get:

$$
\left.\boldsymbol{\Phi}(z)\right|_{|z| \gg \frac{1}{m}}=4 \pi e \int \frac{e^{i k_{\|} z} d k_{\|} d^{2} k_{\perp} /(2 \pi)^{3}}{k_{\|}^{2}\left[1+\frac{e^{3} B}{3 \pi m^{2}} \exp \left(-\frac{k_{\perp}^{2}}{2 e B}\right)\right]+k_{\perp}^{2}} .
$$

It is convenient to integrate over $k_{\|}$closing the integration contour in an upper (lower) semiplane of the complex $k_{\|}$and taking $k_{\|}=i k_{\perp} / \sqrt{1+\frac{e^{3} B}{3 \pi m^{2}} \exp \left(-\frac{k_{\perp}^{2}}{2 e B}\right)}$. In this way we obtain:

$$
\left.\Phi(z)\right|_{|z| \gg \frac{1}{m}}=e \int_{0}^{\infty} \frac{\exp \left[-k_{\perp}|z| / \sqrt{1+\frac{e^{3} B}{3 \pi m^{2}} \exp \left(-\frac{k_{\perp}^{2}}{2 e B}\right)}\right]}{\sqrt{1+\frac{e^{3} B}{3 \pi m^{2}} \exp \left(-\frac{k_{\perp}^{2}}{2 e B}\right)}} d k_{\perp} .
$$


The integral over $k_{\perp}$ converges at $k_{\perp} \lesssim \sqrt{e^{3} B}$, that is why the residue was situated at $k_{\|} \ll m$, where the approximate formula for $P$ we used is valid. At the mentioned values of $k_{\perp}$ the exponent inside the square root can be substituted by one and finally we obtain:

$$
\left.\Phi(z)\right|_{|z| \gg \frac{1}{m}}=\frac{e}{|z|},\left.\quad V(z)\right|_{z \gg \frac{1}{m}}=-\frac{e^{2}}{|z|}-
$$

- the usual Coulomb potential. Strong screening which we obtain in $d=1$ at the distances $|z| \gg 1 / m$ in a realistic case $d=3$ does not occur.

To find a potential at short distances $|z| \ll \frac{1}{m}$ let us substitute $m=0$ in (12):

$$
\begin{aligned}
\Phi(z)|| z \mid \ll \frac{1}{m} & =4 \pi e \int \frac{e^{i k_{\|} z} d k_{\|} d^{2} k_{\perp} /(2 \pi)^{3}}{k_{\|}^{2}+k_{\perp}^{2}+\frac{2 e^{3} B}{\pi} \exp \left(-\frac{k_{\perp}^{2}}{2 e B}\right)}= \\
& =e \int_{0}^{\infty} \frac{\exp \left(-\sqrt{k_{\perp}^{2}+\frac{2 e^{3} B}{\pi} \exp \left(-\frac{k_{\perp}^{2}}{e B}\right)}|z|\right)}{\sqrt{k_{\perp}^{2}+\frac{2 e^{3} B}{\pi} \exp \left(-\frac{k_{\perp}^{2}}{e B}\right)}} k_{\perp} d k_{\perp}
\end{aligned}
$$

Calculating the potential at $|z| \gg 1 / \sqrt{e B}$ we observe that the integral over $k_{\perp}$ is determined by the integrand at $k_{\perp} \ll \sqrt{e B}$. So we took residue at $k_{\|} \approx k_{\perp} \ll \sqrt{e B}$ and the approximate expression for $P$ was used in the domain where it is valid. Performing integration over $k_{\perp}$ we get:

$$
\begin{aligned}
\Phi(z) \mid \frac{1}{m} \gg z \gg \frac{1}{\sqrt{e B}} & =e \int_{0}^{\infty} \frac{\exp \left(-\sqrt{k_{\perp}^{2}+\frac{2 e^{3} B}{\pi}}|z|\right)}{\sqrt{k_{\perp}^{2}+\frac{2 e^{3} B}{\pi}}} k_{\perp} d k_{\perp}= \\
& =\frac{e}{|z|} \exp \left(-\sqrt{\frac{2 e^{3} B}{\pi}}|z|\right), \\
V(z) & =-\frac{e^{2}}{|z|} \exp \left(-\sqrt{\frac{2 e^{3} B}{\pi}}|z|\right) .
\end{aligned}
$$

At the distances which are smaller than Compton wave length we obtain screening of the potential which corresponds to the photon mass $m_{\gamma}^{2}=$ $2 e^{3} B / \pi$. Coulomb potential is screened for the superstrong magnetic fields $B>m^{2} / e^{3}$. 


\section{The energy of the ground state of the hy- drogen atom in the superstrong magnetic fields $B>m_{e}^{2} / e^{3}$}

According to papers [2, 3] in the magnetic fields $B>m^{2} e^{3}$ the ground state energy of the hydrogen atoms equals $E_{0}=-\left(m e^{4} / 2\right) \ln ^{2}\left(B / m^{2} e^{3}\right)$ and at $B=m^{2} / e^{3}$ it equals $E_{\mathrm{cr}}=-\left(m e^{4} / 2\right) \ln ^{2}\left(1 / e^{6}\right)$. For larger magnetic fields the screening of the Coulomb potential at the distances $|z| \lesssim \frac{1}{m}$ occurs. Let us demonstrate that the screening leads to the freezing of the energy - it does not diminish with the growth of the magnetic field.

To find the ground state energy we use the following equation [3]:

$$
E_{0}=-2 m\left(\int_{a_{H}}^{a_{B}} U(z) d z\right)^{2}
$$

We split the integral into two parts: from $1 / m$ to $a_{B}$, where the screening is absent (large $z$ ),

$$
I_{1}=-\int_{1 / m}^{a_{B}} \frac{e^{2}}{z} d z=-e^{2} \ln \left(1 / e^{2}\right)
$$

and from the Larmour radius $a_{H}=1 / \sqrt{e B}$ to $1 / m$, where the screening occurs (small $z)$ :

$$
I_{2}=-\int_{1 / \sqrt{e B}}^{1 / m} \frac{e^{2}}{z} \exp \left(-\sqrt{e^{3} B} z\right) d z=-e^{2} \ln (1 / e) .
$$

Finally we get:

$$
E_{0}=-m e^{4} / 2 \ln ^{2}\left(1 / e^{6}\right)=-m e^{4} / 2 \times 220
$$

and the energy level freezing occurs. The numerical estimates of Shabad and Usov give $73.8 \times 4 \approx 295$ instead of 220 , see Eq. (14) in Phys. Rev. Lett. paper [10].

When $B$ increases further Larmour radius approaches the size of a proton. This happens at $1 / \sqrt{e B} \approx 1 / m_{\rho}, m_{\rho}=770 \mathrm{MeV}, B \approx 10^{20}$ gauss. Taking into account the proton formfactor we get that for larger fields $I_{2}$ does not 
contribute to the energy, factor 220 in (21) should be substituted by 100: the ground level goes up.

Without screening $I=-e^{2} \ln \left(a_{B} / a_{H}\right), E_{0}=-\left(m e^{4} / 2\right) \ln ^{2}\left(B / m^{2} e^{3}\right)$ as it was stated in the beginning of this section.

\section{Conclusions}

The photon polarization operator leads to modifications of the atomic energy levels. The famous example is its contribution to the Lamb shift, the difference of the energies of $2 s_{1 / 2}$ and $2 p_{1 / 2}$ levels of hydrogen. They are numerically small loop corrections to the values of energies determined by the tree level potential. The role of the photon polarization diagram in the superstrong magnetic fields $B>m^{2} / e^{3}=6.2 \cdot 10^{15}$ gauss is qualitatively different. It determines the behaviour of the ground state energy: the formula obtained at tree level becomes invalid and the growth of the coupling energy with $B$ terminates at $B \approx m^{2} / e^{3}$. Screening of Coulomb potential should be more important for the energies of even excited states which are more sensitive to the shape of the potential at small distances [18]. Degeneracy of even and odd excited states in the limit $B \Longrightarrow \infty$ is not lifted by the screening. We study the analogy of the electric potential in $d=1$ QED with massive electrons and in $d=3 \mathrm{QED}$ in strong magnetic fields $B>B_{0}=\mathrm{m}^{2} / \mathrm{e}$ which originates from the coincidence of the polarization operators in these cases. A simple analytical expression which equals the polarization operator with $10 \%$ accuracy enables us to obtain an approximate formula for the electric potential of the point charge in $d=1$ QED with massive fermions and asymptotics of the potential in $d=3$ QED. In $d=1$ QED for a coupling constant $g$ larger than a fermion mass $m$ a tree level formula is modified at $|z|>1 / g$. In $d=3$ QED a tree level formula is modified at the distances

$1 / m>|z|>1 / \sqrt{e^{3} B}$ while at large distances $|z|>1 / m$ Coulomb law is valid.

Analogous results for $D=4$ were obtained in [10].

The other aspect of the Coulomb potential in the strong magnetic field is investigated in paper [19]: it is supposed that fermions obtained their mass due to a magnetic field (dynamical fermion mass).

I am grateful to S.I. Blinnikov, V.A. Novikov, L.B. Okun, V.S. Popov, and A.V. Smilga for useful discussions and to A.I.Rez, who brought to my attention Phys. Rev. publication [10]. 
This work was supported by the grants RFBR 08-02-00494, Nsh-4172.2010.2 and by the contract of the RF Ministry of Science and Education No. 02.740.11.5158.

\section{References}

[1] Landau L.D., Z.Phys., 64 (1930) 629.

[2] Elliott R.J., Loudon R., J. Phys. Chem. Solids 15 (1960) 196; Loudon R., Am. J. Phys. 27 (1959) 649; Wang J.-H., Hsue C.-S., Phys.Rev. A52 (1995) 4508; Lai D., Salpeter E.E., A53 (1996) 152.

[3] Landau L.D., Lifshitz E.M., Quantum Mechanics, Theoretical Physics, v. III - M:, Fizmatlit, 2001, §112, p. 558, problem 3.

[4] Karnakov B.M., Popov V.S., ZhETF 124 (2003) 996.

[5] Loskutov Yu.M., Skobelev V.V., Vestnik MGU, physics, astronomy 24 (1983) 95.

[6] Kuznetsov A.V., Mikheev N.V., Osipov M.V., Mod. Phys. Lett. A17 (2002) 231; Kuznetsov A., Mikheev N., Electroweak Processes in External Electromagnetic Fields (Springer; New York, Berlin, Heidelberg, 2004).

[7] Berestetskii V.B., Lifshitz E.M., Pitaevsky L.P., Quantum Electrodynamics, Theoretical Physics, v. IV - M.: Fizmatlit, 2001, §32, p. 147.

[8] Rabi I.I., Z.Phys. 49 (1928) 507.

[9] Shabad A.E., Usov V.V., Phys. Rev. Lett. 96 (2006) 180401.

[10] Shabad A.E., Usov V.V., Phys. Rev. Lett. 98 (2007) 180403; Phys. Rev. D77 (2008) 025001.

[11] Akhiezer A.I., Berestetskii V.B., Quantum Electrodynamics - M.: Nauka, 1981, Appendix A2, p. 422.

[12] Skobelev V.V., Izvestiya vysshih uchebnyh zavedenii, fizika 10 (1975) 142; Loskutov Yu.M., Skobelev V.V., Phys. Lett. A56 (1976) 151. 
[13] Berestetskii V.B., Lifshitz E.M., Pitaevsky L.P., Quantum Electrodynamics, Theoretical Physics, v. IV - M.: Fizmatlit, 2001, §32, p. 562.

[14] Schwinger J., Phys. Rev. 128 (1962) 2425.

[15] Batalin I.A., Shabad A.E., ZhETF 33 (1971) 483.

[16] Shabad A.E., Ann. Phys. (N.Y.) 90 (1975) 166.

[17] Shabad A.E., Trudy FIAN 192 (1988) 5.

[18] Karnakov B.M., Popov V.S., in print.

[19] Sadooghi N., Sodeiri Jalili A., Phys. Rev. D76 (2007) 065013. 